\title{
STATUS REPORT OF THE SUPERCONDUCTING 5-CELL ACCELERATION STRUCTURE AT CERN
}

P. Bernard, D. Bloess, G. Cavallari, E. Chiaveri, W. Erdt, E. Haebel, H. Lengeler P. Marchand, P. Quéru, J. Tückmantel and W. Weingarten CERN, Geneva, Switzerland

\section{H. Piel}

University of Wuppertal, West Germany

\section{Summary}

At CERN a 5 cell $500 \mathrm{MHz}$ superconducting accelerating cavity has been constructed. At present the cavity is installed in the PETRA storage ring at DESY for a test run which will start middle of March. The cavity is equipped with a high power coupler and with 5 higher order mode couplers, two of the loop type and three of the antenna type. Frequency tuning and regulation are achieved by varying the length of the two end-cells. In a test of the complete cavitycryostat system at CERN, acceleration fields of $2.8 \mathrm{MV} / \mathrm{m}$ and $Q_{0}$-values above $10^{\circ}$ have been reached. The cryostat losses remained below $10 \mathrm{~W}$.

\section{The 5-cell cavity}

The cavicy geometry and the location of the main coupler, of the higher order mode (hom) couplers and of the r.f. probes is shown in fig. 1. Some cavity parameters and experimental results are listed in table 1. The iris opening has been chosen so to obtain a sufficient field flatness with the mechanical tolerances obtainable by our spinning and welding methods described in more detail in ref. 1. The design and the parameters of the main coupler and the hom couplers are described in ref. 2. Each cell is equipped with one hom coupler of either the antenna or loop type. The initially foreseen arrangement ${ }^{3}$ had to be changed because the TMO11 mode, and to a lesser extent the TMi1 mode showed a strongly asymmetric field distribution (due to a slightly different geometry of cell 4). In fig. 1 the new arrangement is shown. In table 2 a few parameters relevant to the hom are given. Before installation the main coupler and all hom couplers were tested in a single cell cavity up to a field level $E_{a c c}=$ $3.6 \mathrm{Mv} / \mathrm{m}$. The field was limited by a cavity quench in no relation with the couplers. Each cell has been equipped with r.f.-(antenna) probes. Their location slightly off-equator allows to couple to all modes.

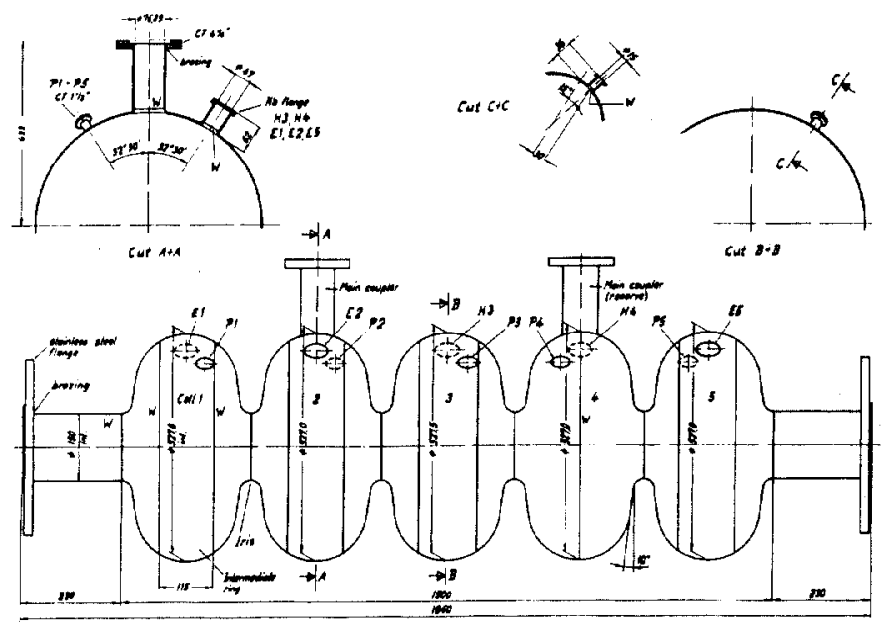

Fig. 1 Layout of 5-cell cavity; H3, H4: coupling ports for hom loop couplers; E1, E2, E5: coupling ports for hom antenna couplers; $\mathrm{PI}-\mathrm{T}^{\mathrm{a}}$. r.f.(antenna) probe ports.
TABLE 1 Some parameters of the $500 \mathrm{HHz} 5$-celi cavity

\begin{tabular}{|c|c|}
\hline $\begin{array}{l}\text { Design frequency ( } \pi \text {-mode) } \\
R / Q \\
E_{p} / E_{a c c} \\
H_{P} / E_{a c c} \\
Q_{0}(C u) \\
\text { Geometry factor } \\
Q_{0} \text { at } 4.2 \mathrm{~K} \text { (measured) } \\
\text { Cavity losses at } 2 \mathrm{MV} / \mathrm{m} \\
Q_{\text {ext }} \text { of main coupler } \\
Q_{\text {ext }} \text { of hom couplers and } R F \\
\text { protes for fundamental mode } \\
\left.2 \text { (f }-f_{0}\right) /\left(f_{\pi}+f_{0}\right) \\
\text { Pressure dependence of frequency } \\
\text { Tuning range }\end{array}$ & $\begin{array}{l}499.665 \mathrm{MHz} \\
665 \mathrm{Ohm} \\
2.05 \\
37.7 \mathrm{G} /(\mathrm{MV} / \mathrm{m}) \\
\sim 47000 \\
272 \mathrm{Ohm} \\
10^{\circ} \\
14 \mathrm{Watt} \\
5.5 \cdot 10^{3} \\
>10^{10} \\
0.97 \\
-90 \mathrm{~Hz} / \mathrm{mbar} \\
>70 \mathrm{kHz}\end{array}$ \\
\hline
\end{tabular}

TABLE 2 Some measured higher order mode parameters for the 5-cell cavity

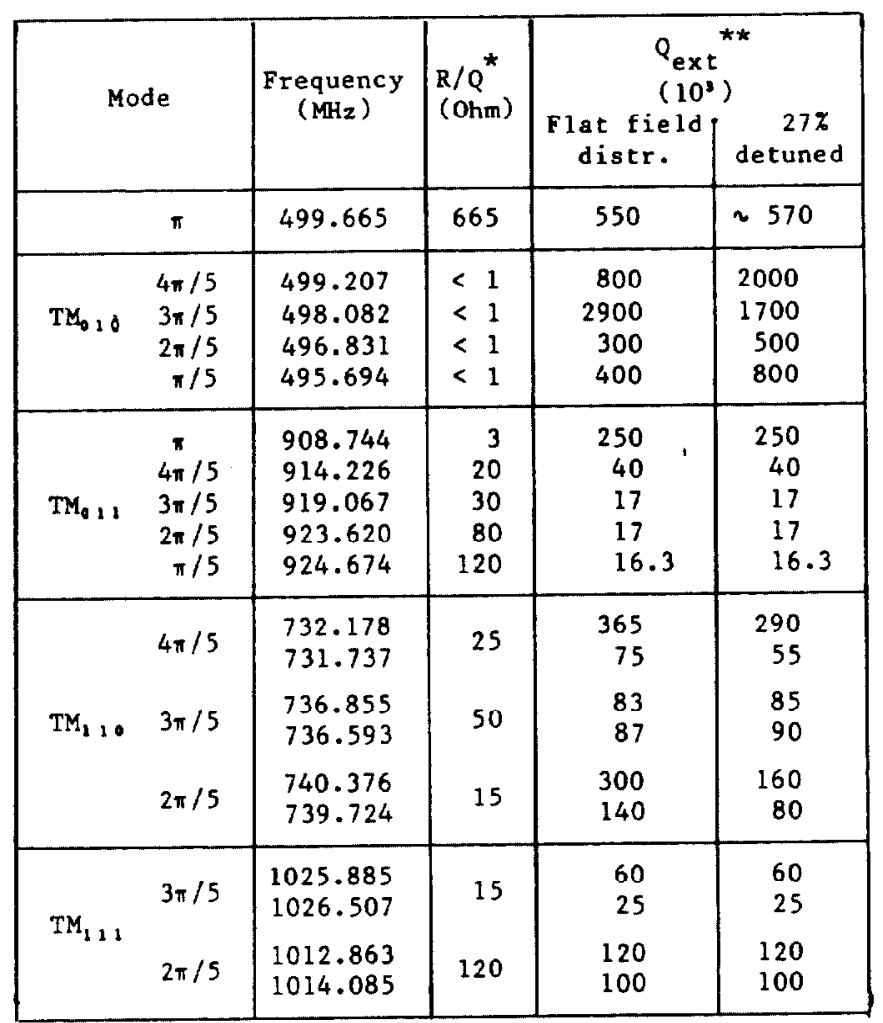

* For flat field distribution; these values change only slightly with $27 \%$ field unflatness except for the TMo1.- $-4 \pi / 5$ mode which increases to $25 \mathrm{ohm}$. For the dipole modes the beam is assumed to be $5 \mathrm{~cm}$ off-axis.

** For the coupler arrangement shown in fig. 1 . 
Each probe has been calibrated for the acceleration mode field amplitude during the cold test of the individual cells. The operation frequency of the acceleration mode was obtained by combining the results of single cell cold tests with Superfish calculations for the 5 -cell cavity. The cavity is mounted inside a support system which allows an individual frequency tuning of the 3 inner cells at room temperature (fig. 2). For the final tuning and for frequency regulation at PETRA, two motor driven mechanical systems allow to change the length of the two end-cells ( $f$ ig. 2 ) independently.

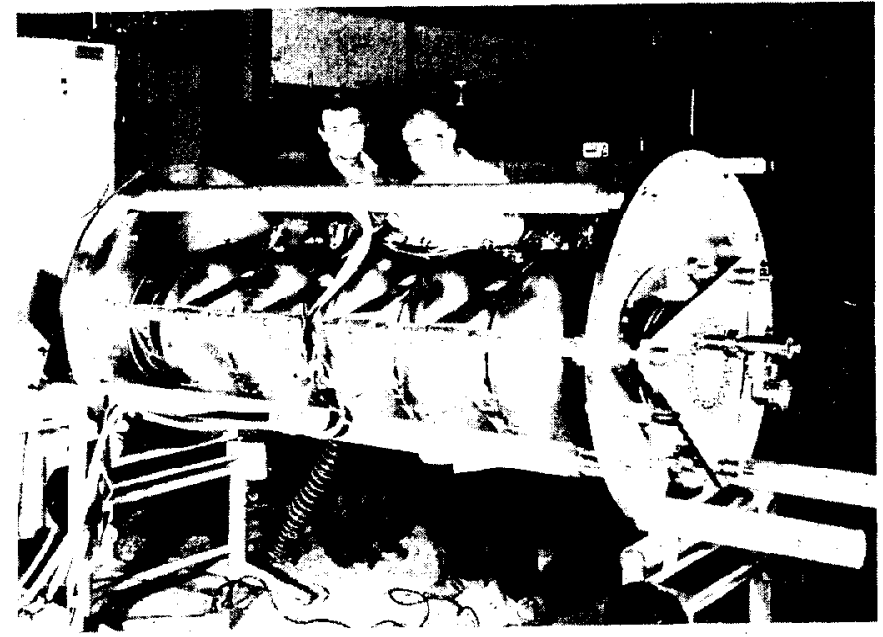

Fig. 2 Photography of the 5-cell cavity with its support and tuning system for the two end-cells. The outer support is used for handling the cavity. Some of the hom couplers and r.f.-probes are visible.

\section{The cryostat}

The cavity is mounted inside an horizontal LHe bath cryostat (fig. 3) leaving ample room around and at both ends of the cavity. The three inner cells with their couplers and r.f. probes remain fully accessible after insertion of the cavity into the He-tank. In order to reduce losses due to heat conduction and r.f. losses along the main 'coupler, the r.f. and control cables and along the beam tubes, counter flows of cold boil' off he gas are used (typical gas flow rate: $0.1 \mathrm{~g} / \mathrm{s}$ ). The evaporation losses of the cryostat with all connections have been measured and amount to $<10 \mathrm{~W}$. The cryostat can be used without major changes for operation with LHe dewars or with a refrigerator. For idling periods it can be kept cold by a $\mathrm{LN}_{2}$ circuit.

\section{Experimental results}

The single cell cavities were tested individually before the final assembly. The results of these tests are presented in ref. 1. Accelerating fields above $5 \mathrm{MV} / \mathrm{m}$ were obtained at the first cooldown and the fields were always limited by a localised quench. The r.f.-losses were dominated by frozen-in magnetic flux $\left(\mathrm{H}_{\text {ext }}=130 \mathrm{mOe}\right)$ and the $Q_{0}-$ values were $\geqslant 10^{9}$.

For the assembly of the 5-cell cavity the 5 cells were welded together at the iris region (fig. 1) from outside. However, because of iris deformations and non-uniform wall thicknesses the initial welds were of poor quality and rewelding aperations had to be done. These weldings were then reground and a (local) chemical polishing of $\sim 50 \mu \mathrm{m}$ at the iris regions was applied.

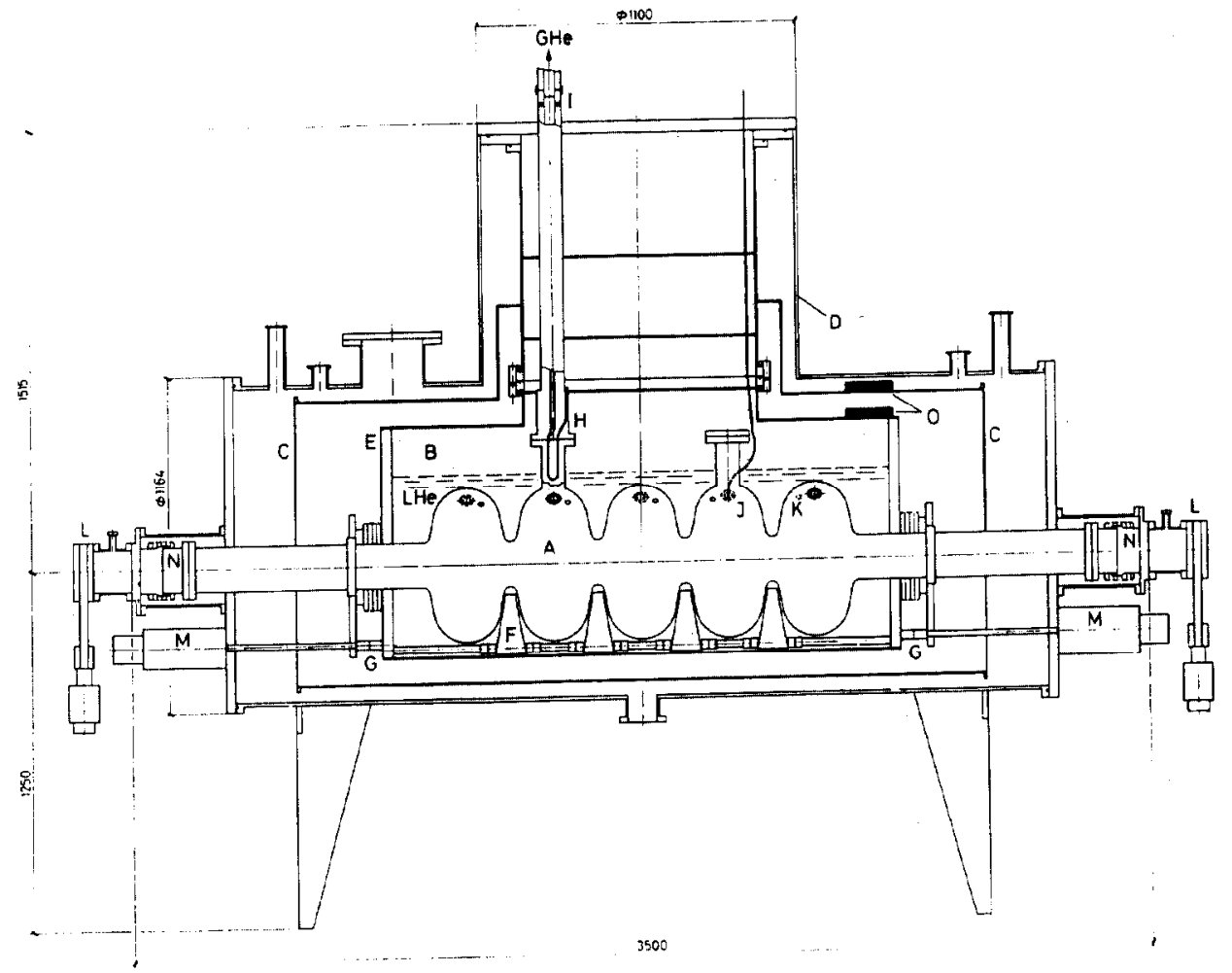

Fig. 3 Layout of the 5-cell cavity inside its cryostat. A: cavity, B: LHe-vessel welded to the covers E, C: cold shield, D: insulation vacuum vessel; $F$ : support and tuning system for inner cells, G: tuner system for cold end-cells ( l low friction screw and 2 normal screws on each side), 0 : supersinsulation. 
In order to check these operations the cavity was measured in a vertical cryostat. For this measurement all hom couplers were mounted (but not the main coupler). After passing many multipactor levels a field $E_{a c c}=2.7 \mathrm{mV} / \mathrm{m}$ was reached. At this field level no quench at the iris regions was observed and it was decided to stop the measurement to avoid a cavity degradation. Because of the relatively low $Q_{0}$ values $\left(\Omega_{0}=6 \cdot 10^{8}\right)$ another rinsing with dust free water was applied before mounting the cavity inside the horizontal cryostat. During this mounting the cavity remained for 6 weeks under protective dust frec gas $\left(\mathrm{N}_{2}\right)$. A leaky joint at the main coupler and a broken r.f. probe in cell 2 were replaced "in situ" by keeping the cavity under $\mathrm{N}_{2}$ gas with a slight overpressure. The cavity was equipped with 40 resistors located around the irises, at the field enhancement regions of the couplers, at the location of the single cell breakdowns and at the bottom of each cell.

The main results of the cold test in the horizontal cryostat were: after tuning of the cavity to the design frequency a field flatness $\left(\bar{E}_{\max }-\bar{E}\right)$ $\overline{\mathrm{E}}=5 \%$ was found. A thermal breakdown affecting a large part of cell 5 was detected at a field level $E_{a c c}=2.1 \mathrm{MV} / \mathrm{m}$. By detuning both end cells in opposite direction an asymetric field distribution with a minimum field in cell $5\left(E_{s}-\bar{E}\right) / \bar{E}=-27 \%$ was established and a mean accelerating field $E=$ $2.8 \mathrm{mV} / \mathrm{m}$ was reached. At this field level a second thermal breakdown at the hom coupler region of cell 3 limited the field $(\stackrel{t}{)}$. As this breakdown occurred in the centre cell a further increase of the mean accelerating field by detuning was not possible. At the maximum fields in the flat and detuned arrangement no electron loading was present in the cells where the quench was observed. The quench field level could be raised by pulsing the $R F$ at a reduced duty cycle. From these observations we conclude that the quench is of thermal origin. Contrary to the 4-cell multicell cavity very little non resonant electron loading was observed in the 5-cell cavity up to the field levels reached. As the coupler ports, however, distort the field configuration of the spherical cavities, we expected some electron multipacting. In fact, multipacting occurred in all single cells and in the 5-cell cavity at distinct field leveis $(1.4,2.5,3.0,4.5 \mathrm{MV} / \mathrm{m})$ which were within experimental errors the same for the different cavities tested. All levels could be processed away after a few hours of $r . f$. processing at the most. In the horizontal test the strong coupling of the main coupler made it particularly easy to pass these levels.
The $Q_{0}$-value at $E_{a c c}=2 \mathrm{MV} / \mathrm{m}$ was determined by the pvaporation rate of LHe. The boil off was then kept constant by substituting the r.f. losses by an electric heating inside the bath so that GHe flow conditions were not changed during the two measurements. By this method one cannot separate the contribution of coupler losses to the evaporation rate but it has been estimated from the measured temperature distributions that these contributions. remain small. From the boil off ( 14 Watt) we estimate $Q_{0}=10^{\circ}$. The external $Q$ of'all hom couplers and $r . f$. probes for the fundamental mode $l i e$ well above $10^{10}$. The external $Q$ of the main coupler and of the arrangement of the 5 hom couplers have been measured, and are 1 isted in table 2 for all hom with $R / Q$ values above $10 \mathrm{ohm}$ and with frequencies below $\approx 1500 \mathrm{MHz}$. It also was checked that a field unflatness of $27 \%$ does change the $R / Q$ and $Q_{\text {ext }} v a l u e s$ only slightly with the exception of the $4 \pi / 5$ fundamental mode (table 2).

The tuner operation and regulation sysfem were checked under various operating conditions. The smallest regulation step is $39.6 \mathrm{~Hz}$ i.e. $n 3 \%$ of the cavity $3 \mathrm{db}$-bandwidth $\mathrm{B}$ of $\mathrm{l} \mathrm{kHz}$. The tuning range obtained with one tuner system exceeds $70 \mathrm{kHz}$ and is thus appropriate for detuning the cavity while idling inside the beam (separation of beam lines for single bunch operation of PETRA: $135 \mathrm{kHz}$ ).

We thank all technicians of our group for their help. We also would like to thank the technicians from the $B E B C, A T$ and cryogenics groups of $E F$ Division as well as all members of $E F$ and $S B$ workshops involved in the programme.

\section{References}

1 P. Bernard et al., CERN/EF/RF 82-9, Internal note (1982).

2 E. Haebel, this conference.

3 P. Marchand and D. Proch, CERN/EF/RF 82-7, Internal Note (1982).

4 K. Halbach and R.F. Holsinger, Part. Acc. 7 (1976) 213.

(*) After these tests we got hints for water residues, produced accicentally during the last rinsing, causing these breakdowns in cell 3 and cell 5. Unfortunately the time schedule for installation in PETRA did not allow us to remove these defects. 